\title{
Consumo de legumbres en Chile. Perspectivas y desafíos
}

\section{Legume consumption in Chile: Perspectives and challenges}

\section{RESUMEN}

La promoción de la alimentación saludable ha sido uno de los pilares en la prevención de las enfermedades crónicas no transmisibles, las que impactan en el aumento de los costos en salud. Es necesario impulsar estilos vida saludable, con medidas estructurales que permitan y faciliten las decisiones más saludables. El consumo de legumbres al menos dos veces a la semana, es promovido a través de las Guías Alimentarias Basadas en Alimentos (GABAs); a pesar de esta recomendación, la tendencia en nuestro país es a disminuir su consumo (tanto el consumo aparente como directo). Para comprender esta práctica es conveniente revisar los elementos que la conforman, entendiendo que la relación entre éstos da lugar a un modo particular de valorar, preparar y comer o no legumbres. Entre las medidas estructurales llevadas a cabo para aumentar el consumo de legumbres en nuestra población, se encuentra su incorporación como ingrediente de algunos de los productos de los Programas Alimentarios, sin embargo, esta medida es insuficiente por si sola para alcanzar la meta planteada en las GABAs. Es necesario implementar estrategias de índole comunicacional para favorecer su valoración social, y difundir innovadoras formas de preparación. Esto debería ir acompañado de medidas de producción agrícola que aumenten su oferta y mejoren su calidad por ejemplo, aumentando la diversidad de especies disponibles. Se requiere un enfoque sistémico, en el cual aspectos estructurales han de encontrar asidero en rutinas cotidianas.

Palabras clave: Legumbres, Chile, Prácticas sociales, Política pública, Guías Alimentarias.

\section{ABSTRACT}

The promotion of healthy eating has been one of the pillars in the prevention of chronic non-communicable diseases, which impact health costs. It is necessary to promote healthy lifestyles with structural measures that allow for and facilitate healthier decisions. The consumption of legumes at least twice a week is promoted through dietary guidelines. Despite recommendations, trends in Chile indicate decreased consumption. To understand this practice it is useful to review the elements related to consumption, understanding that the
Anna Pinheiro, Catalina Ivanovic, Lorena Rodríguez.

Departamento de Nutrición y Alimentos. Ministerio de Salud.

Dirigir correspondencia a: Anna Pinheiro F. Departamento de Nutrición y Alimentos. Ministerio de Salud. Dirección: Monjitas 565. Piso 10. Oficina 1012, Comuna de Santiago, Santiago, Chile. Teléfono: (+56) 225740474. E-mail: anna.pinheiro@minsal.cl

Este trabajo fue recibido el 15 de junio de 2017. Aceptado para ser publicado: 15 de diciembre de 2017.

relationship between elements gives rise to a particular way of valuing, preparing and eating legumes. One structural measure to increase the consumption of legumes in our population has been their incorporation as an ingredient in some of the products in food programs. This measure, however, is insufficient by itself to reach the goal set in the dietary guidelines. It is necessary to implement strategies of a communicational nature to promote their social value and to disseminate innovative ways of preparation. This could be accompanied by measures of agricultural production 
that increase supply and improve quality by increasing the diversity of species available for example. A systemic approach is required, in which structural aspects have to find support in daily routines.

Keywords: Legumes, Chile, Social practices, Public policy, Dietary guidelines.

\section{INTRODUCCIÓN}

La promoción de la alimentación saludable ha sido, en los últimos años, uno de los grandes pilares en la prevención de las enfermedades crónicas no transmisibles. En Chile, los mensajes clave para alimentarse sanamente están contenidos en las Guías Alimentarias Basadas en Alimentos (GABAs) ${ }^{1}$, las que están dirigidas a la población general. Una de dichas guías se refiere a promover el consumo de legumbres al menos dos veces a la semana. A pesar de lo anterior, en las últimas décadas, el consumo de legumbres ha decaído en el país, aunque en estudios sociales aparece aún como una costumbre arraigada en la cultura alimentaria nacional. Es decir es un alimento bien reconocido por todos, pero escasamente consumido.

Considerando la importancia de las legumbres en la nutrición humana, la Organización de las Naciones Unidas para la Alimentación y la Agricultura (FAO) celebró durante el 2016 el "Año Internacional de las Legumbres" el cual reconoce una vez más el elevado valor nutricional de este alimento, su bajo costo, su alta accesibilidad y por ende su importante contribución a la seguridad alimentaria y nutricional. Destacando además de sus beneficios para la salud vinculados a su elevado aporte de fibra, su contribución a mejorar la biodiversidad por su capacidad de aumentar la fertilización de los suelos².

Para avanzar en la incorporación de este grupo de alimentos en la dieta habitual de la población, es necesario, además de fortalecer las acciones de promoción que ya se realizan, abordar el problema desde la perspectiva del ambiente alimentario, entendido como el entorno físico, político, económico y sociocultural en el que vive la población y que influye en sus decisiones de compra y consumo 3 . Por ejemplo en Chile existe la creencia que comer legumbres se relaciona con pobreza, puesto que en periodos pasados de mayor carencia en nuestro país, era la única alternativa para alimentarse ${ }^{4}$.

En este documento expondremos algunos aspectos de la situación de consumo de legumbres en Chile desde la perspectiva de salud y psicosocial, proponiendo algunos desafíos para las políticas públicas.

\section{Enfermedades crónicas no transmisibles}

Las enfermedades crónicas no transmisibles relacionadas a la dieta, tales como obesidad, diabetes, hipertensión y otras, son la principal causa de enfermedad, discapacidad y muerte en Chile y otros países del mundo. Sus causas son multifactoriales y de difícil control. La dieta está multi determinada por distintos factores, individuales (conducta alimentaria) y ambientales (disponibilidad, acceso, precio, valoración social y otros). Las intervenciones que abordan solo los estilos de vida han sido poco exitosas y la mayor parte de las veces resultan ser inadecuadas ${ }^{5}$.

En Chile, de acuerdo a la última Encuesta Nacional de Salud (ENS 2009-2010) ${ }^{6}$, más del 60\% de la población presenta exceso de peso y la obesidad acomete al 25,1\% de los mayores de 15 años. Las enfermedades del sistema circulatorio son la principal causa de muerte en el país y el exceso de peso se perfila como una de las grandes causas de pérdida de años de vida saludable ${ }^{7}$.

Un reciente estudio realizado en población norteamericana determinó que los sujetos obesos presentan un riesgo aumentado en $20 \%$ de morir por cualquier causa de muerte cuando se les compara con sujetos con estado nutricional normal. La mortalidad se adelanta en 3,7 años para cualquier causa y en el caso de las enfermedades cardiovasculares, se adelanta en 5 años para los sujetos con obesidad grado III. Los grupos más afectados son las personas con edades entre 45 años y 64 años para el caso de las muertes causadas por las enfermedades cardiovasculares ${ }^{8}$.

Diversos estudios han podido demostrar el impacto causado por la obesidad y el sobrepeso en el aumento de los costos en salud, tanto directo como indirecto. Withrow y Alter ${ }^{9}$, en una revisión sistemática de los costos directos de la obesidad, estiman, de manera conservadora, que estos pueden variar entre un $2 \%$ al $8 \%$ del gasto en salud. Los autores además estiman que los obesos son $46 \%$ más costosos que las personas con peso normal, tienen $27 \%$ más de consultas médicas y un $80 \%$ más de gastos en medicamentos, lo que implica en una participación en el total del gasto en salud entre el $4 \%$ y $7 \%{ }^{10}$.

En México se estima que para el año 2010 el costo del tratamiento de 30 enfermedades asociadas a la obesidad bordeó a los US\$860 millones y se proyecta un aumento de US\$1,2 billones en 2030 y US\$1,7 billones en $2050^{11}$. En Brasil, se estima que los costos en salud asociados a la obesidad pasarían de US\$ 5,8 billones en 2010 a US\$ 10,1 billones en $2050^{12}$.

En Chile, el estudio de Zarate et al. ${ }^{13}$, determina la influencia de la obesidad en el ausentismo laboral, mostrando que los costos anuales promedio en salud para los trabajadores obesos fue significativamente superior a los trabajadores con estado nutricional normal en un $17 \%$ $(p<0,001), y$ en el caso de los trabajadores con obesidad mórbida $58,0 \%$ superior $(p<0,001)^{13}$. Un reciente trabajo realizado por la Comisión Económica para América Latina y el Caribe indica que la obesidad genera y seguirá generando cada vez más altos costos en salud relacionados con su tratamiento y manejo de complicaciones y secuelas, tales como diabetes e hipertensión, así como también infarto al miocardio, ataque cerebrovascular y algunos tipos de cáncer, entre otras ${ }^{14}$.

En vista de los antecedentes señalados, es ineludible establecer políticas públicas que impacten en la adopción de estilos vida saludable en la población, especialmente en términos de alimentación y nutrición. 
Dieta y prevención de enfermedades crónicas no transmisibles: consumo de legumbres en Chile

Los beneficios para la salud asociados al consumo de legumbres son diversos, tanto por su alto aporte en fibra soluble e insoluble, su buen contenido de proteínas (entre un $20 \%$ y $30 \%$ ), su buena proporción de carbohidratos (entre $50 \%$ y $65 \%$ ) y su bajo aporte de lípidos (cerca de $1 \%)^{15}$; la presencia además entre sus componentes, de otras sustancias como los fitoquímicos y taninos, le otorgan también algunos efectos antioxidantes. Estas características hacen de las legumbres un alimento protector contra el cáncer, las enfermedades cardiovasculares ${ }^{16}$, la diabetes mellitus $^{17}$ y la obesidad ${ }^{18}$.

Las legumbres están presentes dentro de la cultura alimentaria en Chile, aunque su consumo presenta una disminución cuando se evalúan series temporales. Las legumbres más consumidas en el país son las lentejas, porotos, arvejas y garbanzos ${ }^{19}$.

En las últimas décadas, el consumo de legumbres en el país ha ido disminuyendo de acuerdo a datos tanto de consumo aparente como directo. El consumo aparente de legumbres ha disminuido en forma general en una tasa media anual de 2,3\% según datos de la Oficina Nacional de Estudios y Políticas Agrarias (ODEPA), siendo el mayor impacto en el consumo de porotos con una disminución del $28 \%$ entre el 2003 y 2013 (Figura 1) ${ }^{19}$.

El análisis del gasto de los hogares de la Región Metropolita del Gran Santiago y otras capitales del país indica también que la proporción del gasto en legumbres, es mayor en los quintiles inferiores de ingreso ${ }^{20}$.
Por otra parte, la Encuesta Nacional de Consumo Alimentario (ENCA 2010) mostró que la mediana de consumo diario observado fue de $17 \mathrm{~g} /$ día aumentado a 21,8 g/día en zonas rurales y siendo su mayor consumo en la Región Centro-Sur del país (25,9 g/día). En el nivel socioeconómico se presenta un mayor consumo de legumbres (20,6 g/día) que en el nivel socioeconómico alto (17,7 g/día). Mayormente las legumbres son consumidas en preparaciones como guisos; las lentejas y los porotos tipo tórtola son los tipos de legumbres más consumidos por la población ${ }^{21}$.

Para combatir esta situación y para promover el consumo de legumbres, el Ministerio de Salud cuenta con recomendaciones poblacionales de consumo a través de las GABAs las que son actualizadas periódicamente en base a la evidencia, y son difundidas a todos los equipos de salud del país para que sean incluidas en sus acciones de control y tratamiento a través de la consejería en alimentación saludable. El mensaje vinculado a las legumbres es: "Consume legumbres al menos dos veces por semana, sin mezclarlas con cecinas" ${ }^{\prime \prime 1,22}$.

Según la ENCA 2010, solo un poco más del 20\% de la población sigue lo indicado en las GABAs ${ }^{1}$, siendo el mayor cumplimiento entre los niños de 2 años a 5 años (31\%), área rural (33,1\%), zona centro-sur del país (34\%) y en nivel socioeconómico bajo $(29 \%)^{21}$.

\section{Promoviendo el consumo de legumbres a través de los Programas Alimentarios Estatales}

Los Programas Alimentarios Estatales tienen como objetivo la entrega de una alimentación complementaria

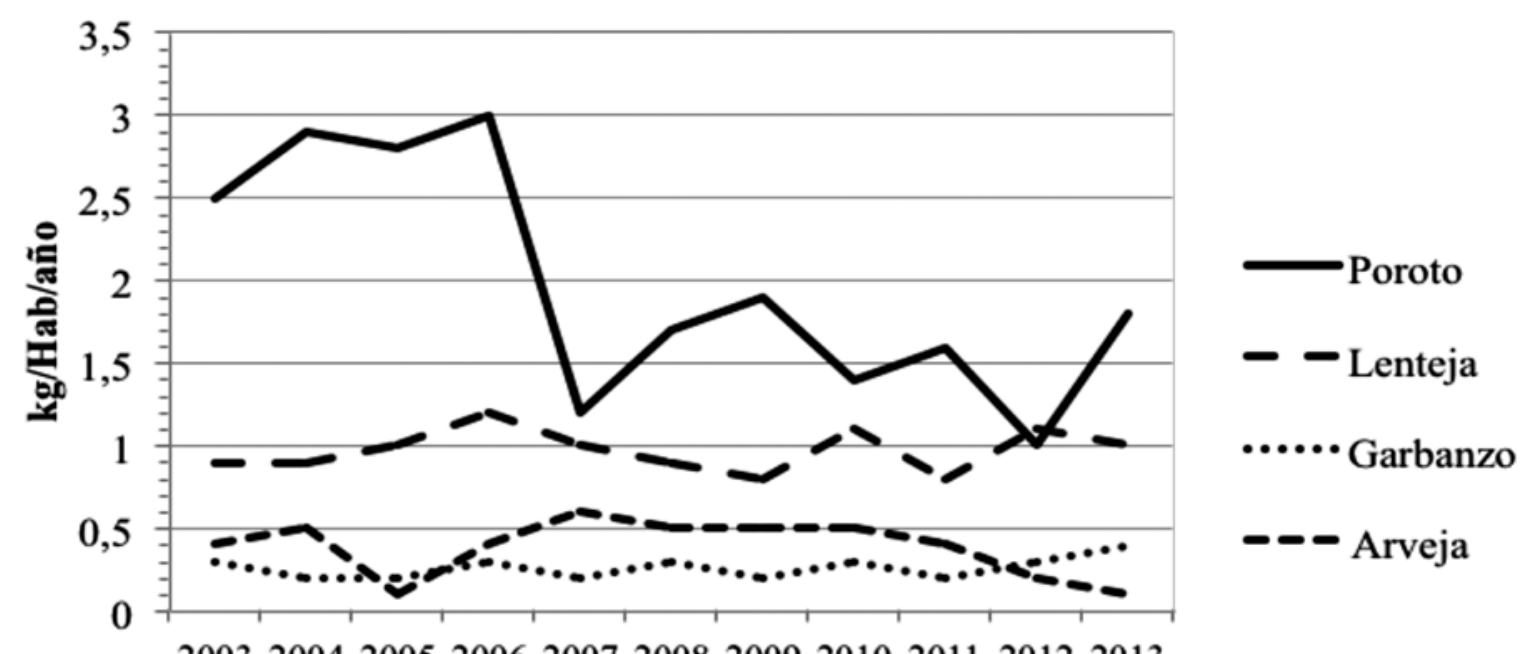

20032004200520062007200820092010201120122013

Años

Figura 1. Consumo aparente de legumbres en Chile: 2003-2013.

Fuente: ODEPA ${ }^{19}$. 
de alta calidad nutricional a distintos grupos vulnerables de la población. Tanto los programas vinculados al Ministerio de Salud como aquellos vinculados al Ministerio de Educación incluyen en distintas formas (preparaciones) y formatos (alimentos in natura, base para sopa crema, etc.) las legumbres, favoreciendo que la población acceda a este alimento en forma gratuita en todo el país.

El Programa Nacional de Alimentación Complementaria (PNAC) y el Programa Nacional de Alimentación Complementaria del Adulto Mayor (PACAM), ambos del Ministerio de Salud tienen como propósito mantener la salud y mejorar el acceso a alimentos sanos y seguros para gestantes, madres que amamantan, niños y niñas menores de 6 años y adultos mayores ${ }^{23}$. Los productos contemplados en estos programas son diseñados de acuerdo a los requerimientos nutricionales de la población beneficiaria. A modo de ejemplo el producto "Mi Sopita" para niños con bajo peso es una sopa crema en base a cereales y leguminosas, con carne y verduras, fortificado con vitaminas y minerales. Su base son las harinas de arroz extruidas (aproximadamente $24 \%$ ), arvejas extruidas (cerca de $17 \%$ ) y maíz extruido (cerca del 14\%). En el año 2015, el volumen distribuido de Mi Sopita fue de $275.300 \mathrm{~kg}$ en todas las regiones del país (2 kg mensuales a cada niño bajo peso en control del país).

Otro ejemplo es el producto "Crema Años Dorados" (CAD), sopa crema con base en cereales y leguminosas, baja en sodio, libre de colesterol y fortificada con vitaminas y minerales. La base de este producto son las harinas de arvejas o lentejas extruidas (cerca de $37 \%$ ), el trigo extruido (aproximadamente 38\%) y el arroz extruido (cerca del 17\%). En el año 2015, el volumen distribuido de Crema Años Dorados fue de $4.673 .540 \mathrm{~kg}$ en todas las regiones del país (1 kg mensual a cada adulto mayor en control de salud). Tanto Mi Sopita como Crema Años Dorados son productos que presentan gran valoración social y muy buena aceptabilidad en los grupos beneficiarios.

El Programa de Alimentación Escolar (PAE) del Ministerio de Educación tiene como finalidad entregar diariamente servicios de alimentación a los alumnos y alumnas en condición de vulnerabilidad de Establecimientos Educacionales Municipales y Particulares Subvencionados del país, en los niveles de Educación Parvulario (Pre-Kínder y Kínder), Básica, Media y Adultos, con el objeto de mejorar su asistencia a clases y contribuir a evitar la deserción escolar.

El PAE ha ido incrementado la frecuencia de entrega de legumbres en los últimos años, acercándose a lo recomendado por las GABAs. Así hoy entregan 2 veces a la semana en algunos subprogramas (Tabla 1). Las legumbres incorporadas en el PAE son porotos, lentejas y garbanzos. En la tabla 1 se presenta un ejemplo de la evolución de las estructuras de las minutas del PAE exigidas en las bases de licitación de la Junta Nacional de Auxilio Escolar y Becas (JUNAEB), del Ministerio de Educación.

Dentro de las preparaciones entregadas en el PAE, las que tiene mayor valoración son aquellas en base a legumbres, presentando una aceptabilidad que supera los $90 \%$. Además, estas preparaciones generalmente son entregadas en cantidades que superan a lo planificado, como son las contribuyendo a un mayor consumo en estos grupos ${ }^{24}$.

\section{Sobre la práctica social en el consumo de legumbres}

Con el objeto de comprender algunas razones que explican el auge y decadencia del consumo de legumbres en Chile, así como encontrar claves socioculturales que podrían favorecer su valoración y consumo, revisaremos las prácticas sociales vinculadas a este tipo de alimentos ${ }^{25}$.

Si se quiere comprender la práctica de comer legumbres, es necesario revisar los elementos que la conforman, entendiendo que la relación entre distintos componentes da lugar a un modo particular de valorar, preparar y comer o no legumbres. En ese sentido un estudio etnográfico obtenido de la observación de 33 sujetos con edades entre los 18 años y 60 años pertenecientes a la clase media santiaguina, en el que se observó su consumo alimentario, mostró una alta densidad simbólica e identitaria en el consumo de legumbres con un periodo de escasez situado algunas décadas atrás, en el cual la restricción alimentaria era relevante y las legumbres constituían uno de los pocos alimentos disponibles. En estas narraciones, las legumbres, ejemplificadas en el poroto, se asociaron a pobreza, escenario opuesto a la relativa abundancia actual. Por lo que estos

\section{Tabla 1}

Ejemplo de variación en la estructura de las minutas exigidas en las bases de licitación del PAE, ítem legumbres, grupo escolar. Período 2004 a 2016.

Año

Licitaciones 2004, 2005 y 2006

Licitación 2008

Licitación 2016
Frecuencia descrita

1 a 2 veces semanal leguminosas

6 veces mensual leguminosas

2 veces por semana considerando almuerzo y cena
Presentación

Guisos

Guisos

Guisos

Elaboración propia considerando las bases de licitación del PAE. 
alimentos están en el imaginario cultural de las personas, pero con asociaciones no siempre positivas ${ }^{26,27}$.

Los relatos de las personas se enlazan con la historia alimentaria de Chile perpetuando una lectura "pobre" de las legumbres, cuyo origen se remonta a las haciendas y el inquilinaje; durante el siglo XIX, la alimentación de los inquilinos estaba marcada por largos periodos en que se consumían sólo guisos como porotos, cazuelas y charqui, mientras que el consumo de carne de vacuno constituía un lujo reservado para las fiestas, como la trilla ${ }^{28}$. Ejemplo de ello es la frase del citado estudio: "Vivíamos en el campo, donde no había nada, donde no había ni azúcar y había que echarle a todo miel, no había un chocolate, no había nada, donde había la cazuela, porotos, lentejas, donde habían las comidas más básicas, donde había un estrechez económica, un apretón salvaje" (varón, C3, Providencia, 38-49 años, soltero, sin hijos, arquitecto independiente) ${ }^{27}$.

Los porotos nos remiten al tiempo de los guisos, a la presencia de una cocinera en casa, quien debía preparar a partir de los productos del campo, platos abundantes que permitieran saciar el hambre de trabajadores a los que la jornada les demandaba un importante gasto energético.

En apenas tres décadas el escenario laboral y alimentario en Chile ha cambiado drásticamente, afectando la práctica de consumir legumbres, cambiando las estructuras sociales y económicas y marcando una nueva relación con el trabajo, la familia y la alimentación. El ascenso social de grupos anteriormente desfavorecidos, se une a la oferta omnipresente y relativamente barata de la industria alimentaria, lo que ha dado lugar a un escenario en que la rutina y ritmos alimentarios se han visto trastocados. Las compras de alimentos se vuelven frecuentes y orientadas por el hedonismo y la falta de tiempo para cocinar, volviendo la ingesta en una instancia relativamente espontánea, por lo que se recurre a comida comprada al paso o platos escasamente elaborados, como hamburguesas, salchichas, o conservas cocidas.

Un ejemplo al respecto del mismo estudio dice: "No hago cosas complicadas que me demanden mucho tiempo, uno tiene que cocinar en una hora y listo, (...) lo soluciono con carne, cualquier cosa", (mujer, C3, Maipú, 50-65 años, divorciada, con hijos, cajera part-time) ${ }^{27}$.

La situación descrita marca el declive de la cocina basada en guisos, lo que afecta particularmente a las legumbres, las que hay que remojar y cocinar por un tiempo más o menos extenso, perdiéndose el ritmo alimentario que las ubicaba en el menú una vez a la semana. Situación que se ve reforzada por la ya citada asociación de las legumbres a la pobreza, valorización que proviene de antaño y se ha perpetuado en la memoria como escasez.

En tiempos de relativa abundancia, las clases medias de ascenso social reciente gozan de poder comer y beber lo que les estaba restringido hace algunas décadas, como la carne de vacuno, las bebidas gaseosas azucaradas, los snacks, pasteles y dulces, frente a los cuales las legumbres tienen poco que ofrecer simbólicamente.
Los adultos y los jóvenes de hoy, en su mayoría, no saben cocinar legumbres, pues no las han visto preparar en casa. La cocina se aprende por medio del gusto y la observación, es una memoria organoléptica que otorga las habilidades y técnicas necesarias para seguir una receta o aventurarse a improvisar un guiso ${ }^{29}$.

\section{Desafíos para aumentar el consumo de legumbres en Chile}

Impulsar el consumo de legumbres es parte de la estrategia de promoción de salud en Chile, en el contexto que la alimentación saludable es uno de los factores protectores más importantes de enfermedades crónicas no transmisibles, las que como ya se ha señalado constituyen el principal problema de salud pública actualmente. En este sentido contar con guías alimentarias nacionales que incluyan entre sus recomendaciones el consumo de legumbres, permite que haya información disponible para toda la población y para su aplicación en distintas acciones de salud, especialmente en atención primaria a través de la consejería en alimentación saludable.

Un importante desafío en este sentido es sensibilizar a los equipos de salud para que esta guía alimentaria sea priorizada y así también instalar la consejería como una herramienta más eficaz para promover el cambio conductual. Esto implica capacitación continua de los equipos de atención primaria y también aumentar y optimizar el tiempo de contacto entre los profesionales y nuestros usuarios y usuarias. Estas medidas constituyen estrategias individuales de cambio de conducta.

Pero también hemos visto que contamos con algunas estrategias estructurales que permiten aumentar el consumo de legumbres en nuestra población, a través de los Programas Alimentarios Estatales que entregan gratuitamente estos productos a un porcentaje importante de niños, niñas, gestantes, adolescentes y adultos mayores.

Sin embargo, son necesarias otras medidas desde el ámbito estructural por ejemplo en el ámbito agrícola, podría ser necesario estimular una producción diversificada de especies promoviendo proyectos de investigación o mediante subvención de pequeños productores agrícolas. En el ámbito de la industria de alimentos puede ser necesaria mayor innovación en atención a disminuir los tiempos de preparación precaviendo los contenidos naturales de nutrientes, pero también innovación en nuevos usos y formas de consumo que aparezcan más modernos y atractivos. En este sentido la colaboración con chefs puede establecer una oportunidad para volver a poner de moda el consumo de legumbres, para reposicionar estos alimentos en nuestra sociedad.

Reconociendo que la decadencia del consumo de legumbres es sintomática de la aceleración de los tiempos en nuestra sociedad y del desarrollo de un nuevo modo de vida reñido con los ritmos "rurales" de las legumbres, es necesario instalar algunas prácticas familiares de distribución de tareas, de organización de compras y de 
plan de menú semanal que permita incorporar en la dieta familiar alimentos que requieren mayor preparación. La reciente campaña del Ministerio de Salud "El Plato de tu Vida" entrega algunos consejos, tips, ideas y recetas que pueden contribuir con este cambio de práctica familiar (www.elplatodetuvida.minsal.cl).

Considerando los aspectos psicosociales y culturales de la alimentación, se puede retomar la valoración familiar histórica de las legumbres, aprovechando la carga afectiva que guardan en nuestra memoria, así como su materialidad por medio de objetos y formatos de preparación que permitan volver a traer estos guisos a nuestros tiempos, y a difundir de generación en generación los conocimientos tan necesarios para su preparación. Para esto podría ser un desafío interesante generar espacios de reflexión y eventualmente una campaña comunicacional intersectorial con el objetivo de rescatar a las legumbres desde nuestra cultura alimentaria ancestral, sin desconsiderar la aceleración del mundo actual. Para sostener esta práctica, se exige un enfoque sistémico, en el cual los aspectos estructurales han de encontrar asidero en las rutinas cotidianas de los distintos sujetos.

El desafío es necesariamente intersectorial e interdisciplinario, pues debe considerar aspectos individuales, estructurales y del entorno que permitan relegitimar y viabilizar una práctica social que ha caído en algún grado de desuso producto de un cambio social mayor, pero juega a nuestro favor una historia alimentaria en que las legumbres gozaron de gran importancia y valoración.

\section{BIBLIOGRAFÍA}

1. Ministerio de Salud. Subsecretaria de Salud Pública. Informe final "Estudio para revisión y actualización de las guías alimentarias para la población Chilena" [Internet]. 2013. Available from: http://www.minsal.cl/portal/url/item/dde0bc471a56a001e0400 10165012224.pdf

2. FAO. Conclusiones del Año Internacional de las Legumbres [Internet]. 2017 [cited 2017 May 3]. p. 1. Available from: http://www.fao.org/zhc/detail-events/es/c/470558/

3. Swinburn B, Sacks G, Vandevijvere S, Kumanyika S, Lobstein T, Neal B, et al. INFORMAS (International Network for Food and Obesity/non-communicable diseases Research, Monitoring and Action Support): overview and key principles. Obes Rev 2013; Suppl(1): 1-12.

4. Rioseco R, Egaña D, Galvez P, Masferrer D. Marco conceptual sobre los factores condicionantes de los ambientes alimentarios en Chile [Internet]. Santiago, Chile; 2016. Available from: http:// www.bibliotecaminsal.cl/marco-conceptual-sobre-los-factorescondicionantes-de-los-ambientes-alimentarios-en-chile/

5. Yach D, Hawkes C, Gould C, Hofman K. The global burden of chronic diseases: overcoming impediments to prevention and control. JAMA 2004; 291(21): 2616-2622.

6. Minsal. National Health Survey ENS 2009-2010 [Internet]. 2009. Available from: http://epi.minsal.cl/wp-content/uploads/2012/07/ Informe-ENS-2009-2010.-CAP-5_FINALv1julioccepi.pdf

7. Minsal. Informe final estudio de carga de enfermedad y carga atribuible [Internet]. 2008. Available from: http:// epi.minsal.cl/epi/html/invest/cargaenf2008/Informe final carga_Enf_2007.pdf
8. Borrell L, Samuel L. Body Mass Index Categories and Mortality Risk in US Adults: The Effect of Overweight and Obesity on Advancing Death. Am J Public Health 2014; 104(3): 512-519.

9. Withrow $D$, Alter D. The economic burden of obesity worldwide: a systematic review of the direct costs of obesity. Obes Rev 2011; 12(2): 131-141.

10. Finkelstein E, Trogdon J, Cohen J, Dietz W. Annual medical spending attributable to obesity: payer-and service-specific estimates. Heal Aff [Internet]. 2009; 28(5): w822-831. Available from: http://content.healthaffairs.org/content/28/5/w822.full. pdf $+h t m l$

11. Rtveladze K, Marsh T, Barquera S, Sanchez Romero L, Levy $D$, Melendez G, et al. Obesity prevalence in Mexico: impact on health and economic burden. Public Heal Nutr. 2013; 1:1-7.

12. Rtveladze K, Marsh T, Webber L, Kilpi F, Levy D, Conde W, et al. Health and economic burden of obesity in Brazil. PLoS One. 2013; 8(7):e68785.

13. Zarate A, Crestto M, Maiz A, Ravesta G, Pino M, Valdivia G, et al. Influencia de la obesidad en los costos en salud y en el ausentismo laboral de causa médica en una cohorte de trabajadores. Rev Med Chile. 2009; 137(3):337-344.

14. Fernández A, Martinez R, Carrasco I, Palma A. Impacto social y económico de la malnutrición Modelo de análisis y estudio piloto en Chile, el Ecuador y México [Internet]. 2016. Available from: http://repositorio.cepal.org/bitstream/handle/11362/ 41247/4/LCTS2017-32_es.pdf

15. Mudryj A, Yu N, Hartman T, Mitchell D, Lawrence F, Aukema H. Pulse consumption in Canadian adults influences nutrient intakes. Br / Nutr. 2012; 108(Suppl 1):S27-36.

16. Mudryj A, Yu N, Aukema H. Nutritional and health benefits of pulses. Appl Physiol Nutr Metab. 2014; 39(11):1197-1204.

17. Ramdath D, Renwick S, Duncan A. The Role of Pulses in the Dietary Management of Diabetes. Can I Diabetes. 2016; 40(4):355-363.

18. Marinangeli $C$, Jones $P$. Pulse grain consumption and obesity: effects on energy expenditure, substrate oxidation, body composition, fat deposition and satiety. Br J Nutr. 2012; 108(Supp/ 1):S46-51.

19. Ministerio de Agricultura. Consumo aparente de principales alimentos en Chile, Oficina de Estudios y Políticas Agrarias [Internet]. Santiago, Chile. 2012. Available from: http://www. odepa.gob.cl/odepaweb/publicaciones/doc/7004.pdf

20. Araneda J, Pinheiro A, Rodriguez L, Rodriguez A. Consumo aparente de frutas, hortalizas y alimentos ultraprocesados en la población chilena. Rev Chil Nutr. 2016; 43(3):271-8.

21. Ministerio de Salud. National Survey of Food Consumption (ENCA) [Internet]. Available from: http://web.minsal.cl/sites/ default/files/ENCAINFORME_FINAL.pdf

22. Olivares S, Zacarías I, González C, Fonseca L, Rivera L. Estudio para la construcción de una imagen gráfica didáctica que ilustre la información contenida en las guías alimentarias para la población chilena [Internet]. 2014. Available from: http://web.minsal.cl/sites/default/files/files/Estudio para la construcci\%25C3\%25B3n de una imagen gr $\% 25$ C3\%25A1fica did $\% 25$ C3\%25A1ctica que ilustre la informaci\%25C3\%25B3n contenida en las Gu\%25C3\%25ADas Alimentarias para la poblaci\%25C3\%25B3n chilena_MINSAL 2015.pdf

23. MINSAL. Technical Standard of Food Programs [Internet]. Chile; 2016. Available from: http://web.minsal.cl/wp-content/ uploads/2015/09/2016.04.20-Norma-Técnica-Prog.-Alimentariosaprobada-por-Jurídica.pdf

24. Barrios L, Bustos Z N, Lera M L, Kain B J. Raciones de desayuno 
y almuerzo entregadas a preescolares por el Programa de Alimentación Escolar (PAE): calorías aportadas y consumidas. Rev Chil Nutr. 2013; 40(4):330-5.

25. Reckwitz A. Toward a theory of social practices. A development in culturalist theorizing. Eur J Soc Theory. 2002; 5(2):243-63.

26. Shove E. Everyday practice and the production and consumption of time. In: Shove E, Trentmann F, Wilk $R$, editors. Time, consumption and everyday life: Practice, materiality and culture. Oxford, Reino Unido: Berg Publishers; 2009. p. 17-34.
27. Ivanovic C. Significados del consumo en las clases medias santiaguinas. Tesis para optar al grado de doctor en Sociología. Universidad Alberto Hurtado, Santiago, Chile; 2014.

28. Orlove B. Beyond Consumption: Meat, Sociality, Vitality and Hierarchy in Nineteenth Century Chile. In: Friedman J, editor. Consumption and Identity. Londres, Inglaterra: Harwood Academic Publishers; 1994.

29. Ivanovic C. La Cocina: un espacio para la puesta en escena de las identidades de género de los(as) habitantes de Punta Arenas. Universidad de Chile; 2008. 